\title{
Serious Games Adaptation According to the Learner's Performances
}

\author{
Amine Belahbib, Lotfi El Aachak, Mohamed Bouhorma, Othman Bakkali Yedri, Slimani Abdelali, \\ Elouaai Fatiha \\ Computer Science, Systems and Telecommunication Laboratory (LIST), Faculty of Sciences and Technologies, \\ University Abdelmalek Essaadi Tangier, Morocco
}

\section{Article Info \\ Article history: \\ Received Aug 28, 2016 \\ Revised Oct 26, 2016 \\ Accepted Nov 10, 2016}

\section{Keyword:}

Adpatation

E-learning

Multi-Agents system

Neural Network

Serious Games

\begin{abstract}
Basically, serious games provides enjoyment and knowledge, several researches in this field have focused into joining these two proprieties and make the best balance between them, in order, to provide the best game and enjoyable game experience and ensure the learning of the needed knowledge. Players differ and their knowledge background can be a lot different from one to the other. This study focused on how the SG adapts and provide the needed knowledge and enjoyment. The game should analyze players behavior from different angles, thus it can add difficulty, information, immersion or enjoyment modules to fit the player skills/knowledge.
\end{abstract}

Copyright @ 2017 Institute of Advanced Engineering and Science. All rights reserved.

\section{Corresponding Author:}

Amine Belahbib,

Computer Science, Systems and Telecommunication Laboratory (LIST),

Faculty of Sciences and Technologies,

University Abdelmalek Essaadi Tangier, Morocco.

Email: amine.belahbib@gmail.com

\section{INTRODUCTION}

Learning through serious games has become one of the most popular teaching methods used to transfer both knowledge and skills to the learners, in several fields. The use of this kind of video games may even lead to superior learning compared with traditional instructional methods [1], [2]. Despite the effectiveness of these tools in the field of education, several problems related to understanding and motivation can happen, related to the capacity and the level of learning which differs from a learner to another. Indeed, the adaptation of such video games according to the learner performance can be beneficial, and can resolve the several encountering problems.

The adaptation of serious games can impact several elements like the game world and its varied objects, the layout of the game world can be made simpler for underachieving players [3]; the game play mechanics by adjusting the difficulty to provide player aim assistance, according to individual skills [4]; the attributes of the non-player characters in the game based on the artificial intelligence algorithms [5], the game storytelling by adapting the sequence of events to the pace or the behavior of the player [6] ; and the flow of events and actions during the game sequence by monitoring the players' actions and based on that certain points in the plot are included in the game [7].

The process of the study consists in the first step to analyze serious games already on the market and break it into skills/knowledge proprieties; then as a second step, to study several serious games and extract all the proprieties Table 1 that can be adjusted by using several learning algorithms. 
Table 1. Knowledge and Game Skills Properties

\begin{tabular}{ll}
\hline Knowledge & Gaming skills \\
\hline Observation & Speed \\
Logic & Duration \\
Memory & Endurance \\
Manage & Precision \\
& Decision Making \\
\hline
\end{tabular}

The nature of these proprieties leads us to separate knowledge from Gaming skills, this is said, the necessity to make three levels for each propriety (easy, normal, and hard). Knowledge levels will rely on the rate of use and showing messages / graphics to inform the player. On the other hand gaming skills will rely on the level of difficulty or rate of appearance in the game.

Assuming that the game will follow one specific scenario which should be accurate and rich in term of graphics, we can make the game adapt each of the said proprieties according to the available levels to fit the learner knowledge and skill. To make it simple, we have specified just three levels in each property, but nothing stops us from making the levels being a percentage or other specific number of levels.

The current paper presents a serious game called EQUAFUN, which teaches the students how to solve mathematical equation in an easy and fun way, the proposed serious game is equipped with a system of adaptation based on the learners' performances, in order, to adapt the game according to the level of the student. A discussion of the obtained result with the future research work in the field will conclude this paper.

\section{RELATED WORK}

According to an experiment conducted in the paper: Player Performance, Satisfaction, and Video Game Enjoyment [8], it shows how the difficulty can affect the satisfaction of the player, as a result, too easy games leads to boredom and too hard games may lead to frustration. Based on these results we take into consideration the player profile, players that might have different levels of knowledge and gaming skills. However, in this study, we focused on 5 attributes, resulting the adaptation of three game skills.

\subsection{Player Satisfaction}

According to an experiment conducted in the paper: Player Performance, Satisfaction, and Video Game Enjoyment [8], it shows how the difficulty can affect the satisfaction of the player, as a result, too easy games leads to boredom and too hard games may lead to frustration. Based on these results we take into consideration the player profile, players that might have different levels of knowledge and gaming skills. However, in this study, we focused on 5 attributes, resulting the adaptation of three game skills.

\subsection{Artificial Intelligence}

According to the study Rapid Adaptation of Video Game [9], AI Difficulty scaling is one of the main elements that should be adaptable using AI, but talking about the difficulty is quite large as a concept, and again the background/profile of the player is the main repair to know if the game is easy/difficult. In this paper, we focused on the player, in order to get his intellectual level to adapt the game accordingly and in addition, the game should be able to learn and collect information from players the more games are played.

\section{SERIOUS GAME ADAPTATION}

The main objective of this current paper is to adapt a serious game based on several parameters related to the learner behaviors during the game and the adaptation will be done by using a variety of machine learning algorithms, e.g. "neural network, decision tree", the description of the proposed serious game and the establishment of the hall system will be described in this section.

\subsection{The Propsed Serious Game "EQUAFUN"}

The following game "Figure.1" presents the adaptation approach, the game is based on five of the basic attributes (Age, Sex, Average of response time, Number of wrong answers, Number of redundant faults) in order to produce the adapted game. The goal of the game is to teach kids how to solve simple equation by using a balance and fruits, the player will have one unknown bag and other types of fruits, it is a kind of representation of equations like: $a x+b=0$. The player should drag the fruits from one side to another until they deduce the weight of the unknown bag. 

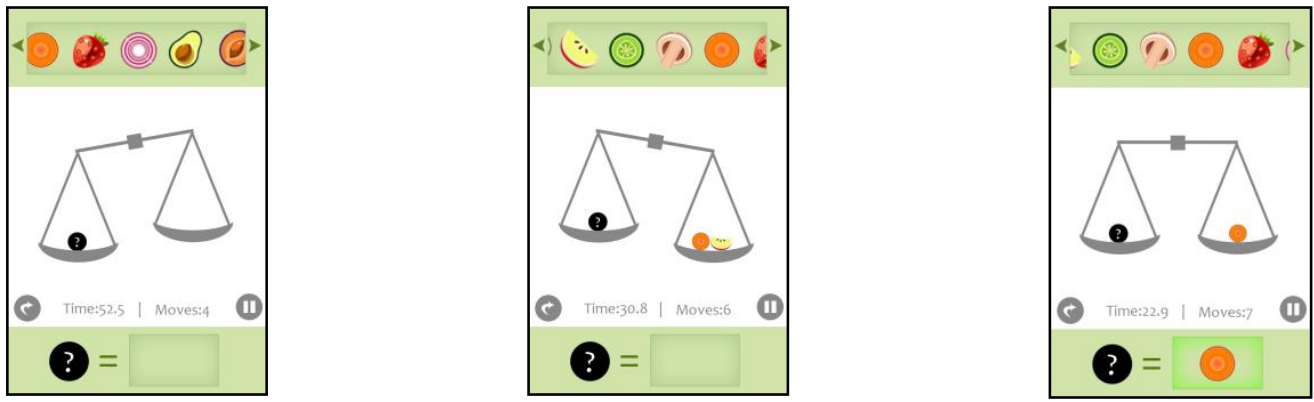

Figure 1. Screenshots from the EQUAFUN Serious Game

These figures show the first level where the player tried two fruits, "orange/apple", but the game still unbalanced, the player moved away the apple and left the orange, this result a correct balance. This way the player knows that the unknown bag contains an orange, so he should drag it to the result on the bottom of the screen.

The game adapts according these basic attributes devided to two types, Constant attributes (Age, Sex) and Dynamic attributes (Average of response time, Number of wrong answers, Number of redundant faults). These attributes are chosen according to the gameplay and type of game, in our case we took all the attributes present in this game.

The said attributes are used in a way to adapt and change the game content according to the player needs, in order to to make the relation between the player input and the game output, our adapted game relies on these deduced three propreties: logic, memory and speed. The deduction of each proprity is done using some of the basic attributes taken as entrance while the player is playing the game:

\section{Average of response time $=>$ Speed}

If the player is slow in resolving levels we will add more allowed time in the next levels before decreasing it further in the game advancement.

The Number of wrong answers $=>$ Logic

If the player makes a lot of errors based on logic, the game will try to advance slowly in logic difficulty, adding other parallel balances.

\section{Number of redundant faults $=>$ Memory}

If the player is making a lot of redundant errors the game should slow down the advancement and try to show more redundant problems using more fruits.

As result, while game is played, these propreties (Speed, Logic and Speed) will change to fit the level of the player, the game itself will rely on the level of each of the proprities in order to provide the adapted game that fit the player's current level.

\subsection{System Architecture}

As mentioned above the hall system is based on a multi-agents system MAS. The MAS is composed of several entities called intelligent agents connected each other in order, to solve problems that are difficult or impossible for an individual agent.

As show in the Figure 2, the architecture of the proposed system is divided on two principal parts, the first part represents an application server where the proposed serious game is deployed. The actions and learner's behaviors are saved in a database, in order, to be analyzed or used by the agents later. By cons the second part is established by using JADE [10].

The proposed MAS, is composed of two agents. The first one is the predictor agent, its role is to predict the player performance during the game sequence, and this agent is established by using artificial neural network. The second one is the decision maker, it takes a decision to adapt the game according to both learning level and performance of the player, there is a communication between the two agents the predictor evaluates the learners and sends this information into decision maker that adapts the game, and all of these operations are made in real time. 


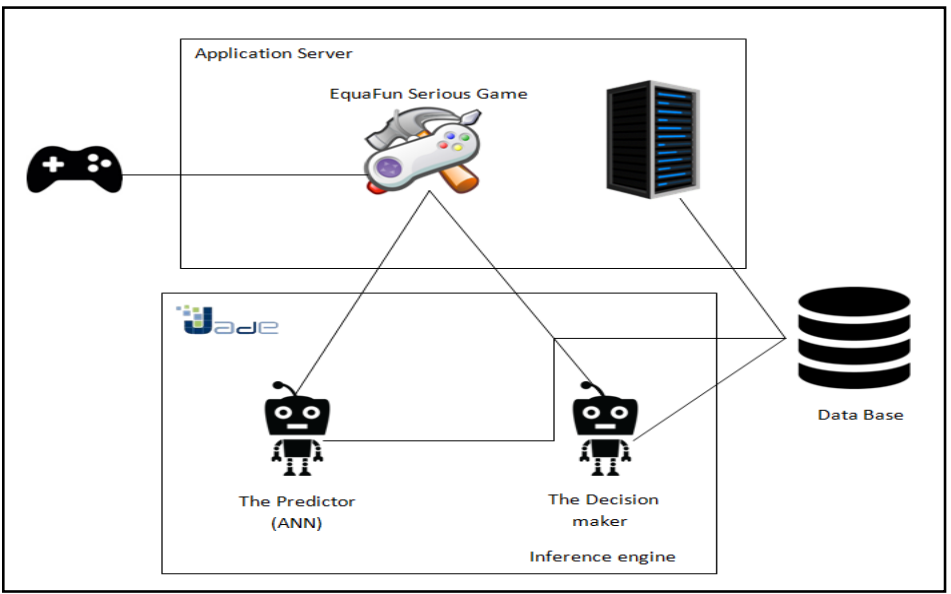

Figure 2. MAS Architecture for the propsed serious game

The information saved into the database, are show in the Table 2, these information will be operated also by the tools of learning analytics and educational data mining, to have a global view on the progression of all the learners.

Table 2. Database Attributes

\begin{tabular}{l}
\hline \multicolumn{1}{c}{ Knowledge } \\
\hline Age \\
Sexe \\
Average of response time \\
Number of wrong answers \\
Number of redundant faults \\
\hline
\end{tabular}

\subsection{The Predictor Based on Artificial Neural Network}

The predictor is an agent of MAS that predicts the learners' performances during a game sequence, this current agent is established by using one of the learning machine algorithm called neural network NN, the $\mathrm{NN}$ is a field of Artificial Intelligence that allows finding the data structures and algorithms for learning regression, and classification of data, by inspiration from the human brain. It has been developed as generalizations of mathematical models of biological nervous systems. A first wave of interest in neural networks emerged after the introduction of simplified neurons [11].

The signal flow from the inputs is considered to be in one direction. The neuron output signal $\mathrm{O}$ is given by the following relationship described below:

$$
O=f(n e t)=f\left(\sum_{i=0}^{n} w i * x i\right)
$$

where $\mathrm{Wi}$ is the weight vector, and the function $\mathrm{f}$ (net) is referred to activation function. The variable net is defined as a scalar product of the weight and input vectors.

net $=\mathrm{wT} \mathrm{x}=\mathrm{w}_{1} \mathrm{x}_{1}+\mathrm{w}_{2} \mathrm{x}_{2}+\ldots+\mathrm{w}_{\mathrm{n}} \mathrm{x}_{\mathrm{n}}$

where $\mathrm{T}$ is the transpose of a matrix, the output value $\mathrm{O}$ is computed as:

$O=f($ net $)=\left\{\begin{array}{l}1, \text { if } w T>\theta \\ 0, \text { otherwise }\end{array}\right.$

where $\theta$ is called the threshold level; and this type of node is called a linear threshold unit. 
The NN architecture "Figure 3" of the predictor agent is composed of three layers "input, hidden and output layers", in each layer there is a node called Bias. The characteristic of the NN architecture is detailed in Table 3.

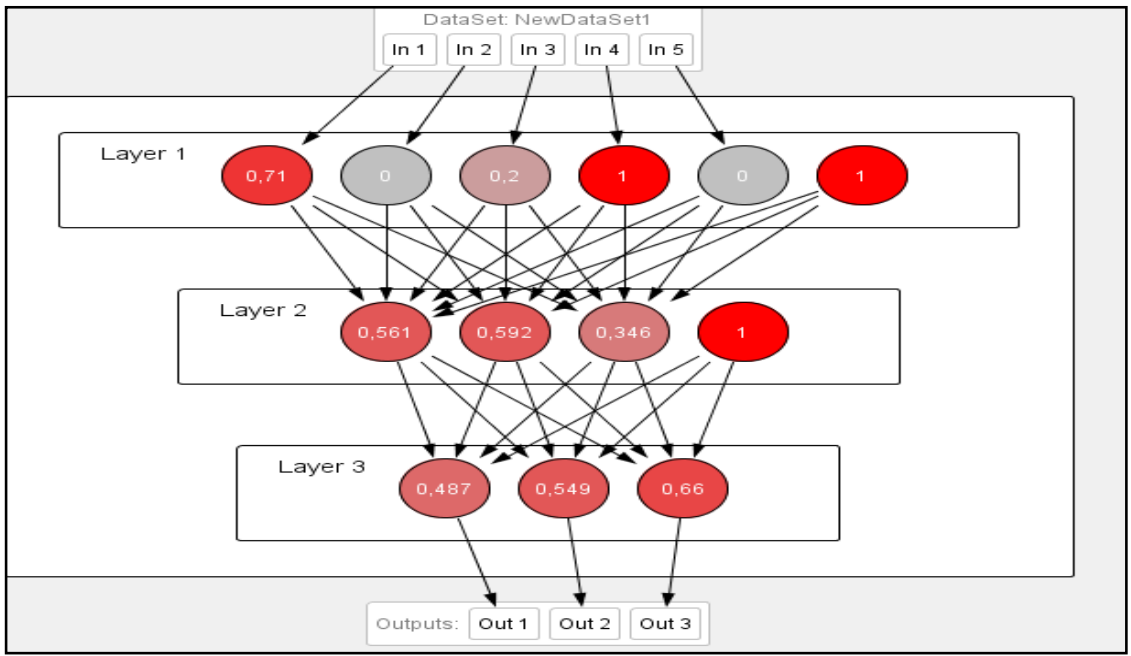

Figure 3. Neural Network Architecture for the Predictor

Table 3. Database Attributes

\begin{tabular}{cc}
\hline Element & Value \\
\hline Input layer & 5 nodes \\
Hidden layer & 3 nodes \\
Output layer & 3 nodes \\
Activation function & Sigmoid \\
Learning algorithm & Resilient back propagation \\
Training error Threshold & 0.001 \\
Learning rate & 0.1 \\
\hline
\end{tabular}

As described in the table above, there are 5 nodes (age, sex, Average of time response, number of wrong answers and number of redundant faults) in input layer, five nodes in the hidden layer and three output nodes those nods have three values ( $\mathrm{G}, \mathrm{M}$ and $\mathrm{B}$ ), which are good, Medium and bad, the activation function is a sigmoid for each node by cons the learning algorithm is the resilient back propagation [12], the chosen algorithm has advantages in training speed and efficiency.

\subsection{The Decision Maker Based on Descion Tree}

The second agent in the proposed system is called the decision maker; its role is to take decisions, in order, to adapt the serious game according to several parameters like the result of the predictor agent "calculated through the neural network", the number of wrong answers, the average of the time taken by the learner and the number of the redundant faults, all these parameters will build dynamically a decision three [13].

The decision tree is formalism for expressing such mappings and consists of nodes linked to several sub-trees and leafs or decision nodes labeled with a class which means the decision.

The C4.5 [13] is the learning algorithm that will generate the three according to the data saved in the database; the proposed algorithm is an extension of ID3 algorithm; it builds decision trees from a set of training data in the same way as ID3 by using the concept of information entropy, the algorithm of C4.5 for building the decision tree is described below:

1. Check for base cases

2. For each attribute a: Find the normalized information gain ratio from splitting on a.

3. Let a_best be the attribute with the highest normalized information gain.

4. Create a decision node that splits on a_best.

5. Recur on the sublists obtained by splitting on a_best, and add those nodes as children of node. 
The Figure 4 presents an example of the decision tree generated dynamically from the data saved in the database and with the use of C4.5 learning algorithm.

The attributes "Inputs" that feed the decision tree are: the result of the predictor agent "calculated through the neural network, according to the learner's performance", the number of wrong answers, the average of the time taken by the learner and the number of the redundant faults. By cons the classes "Outputs" are: logic, reflex, memory and speed

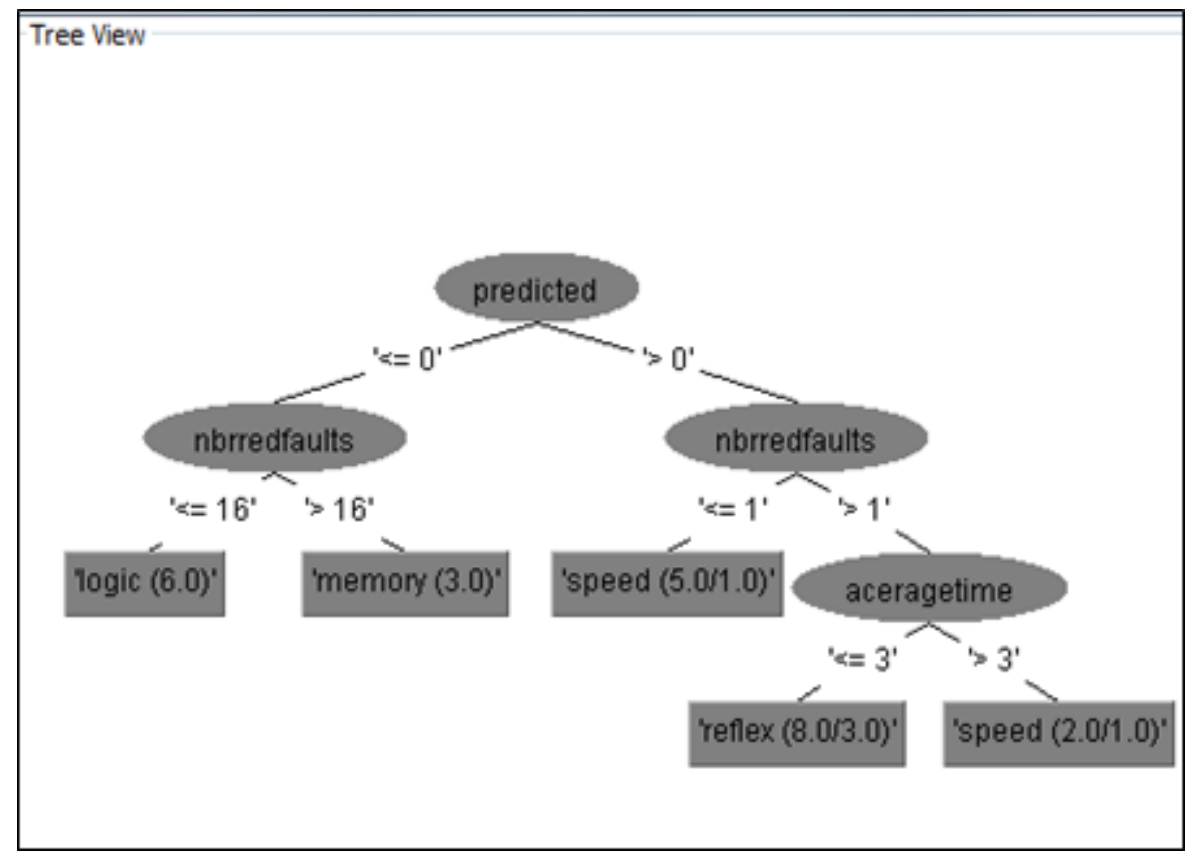

Figure 4. The Decision Tree of the Decidor

The obtained results will be interpreted and discussed in the next section, in order to evaluate the impact of the adaptation of the learning process through serious games.

\section{RESULT AND DISCUSSION}

Two versions of the game have been made, the one that provides all the levels and variance, and the second game using the adaptation, which takes into account the inputs in order to adapt the proposed serious game accordingly. The second version is learning from the players and has its own learning process, that's why we will cluster the players into 3 groups:

a. Group1: Playing normal game $(\mathrm{N}=90)$

b. Group2: Playing adaptive game $(\mathrm{N}=90)$

c. Group3: Playing experienced adaptive game $(\mathrm{N}=50)$

This clustering should give us the big image on what is the impact of this game adaptation and how it evolves by the time to fit the player needs based on their profile.

As mentioned before the adaptation of the proposed serious game is based on two machine learning algorithms, the neural network "Agent 1"and the decision tree "Agent 2", the first one will predict the learner's performance during the game sequence, by cons the second one will modify one of the four parameters according to the result given by the neural network and other information collected during the game.

During the passage from one scene to another, all the collected parameters will feed the predictor agent, this one will feed the decision making agent by the predicted performance, afterwards the decision making agent will modify the chosen parameter of "EquaFun" game, according to this automated modification, the game will be adapted Figure 5. 

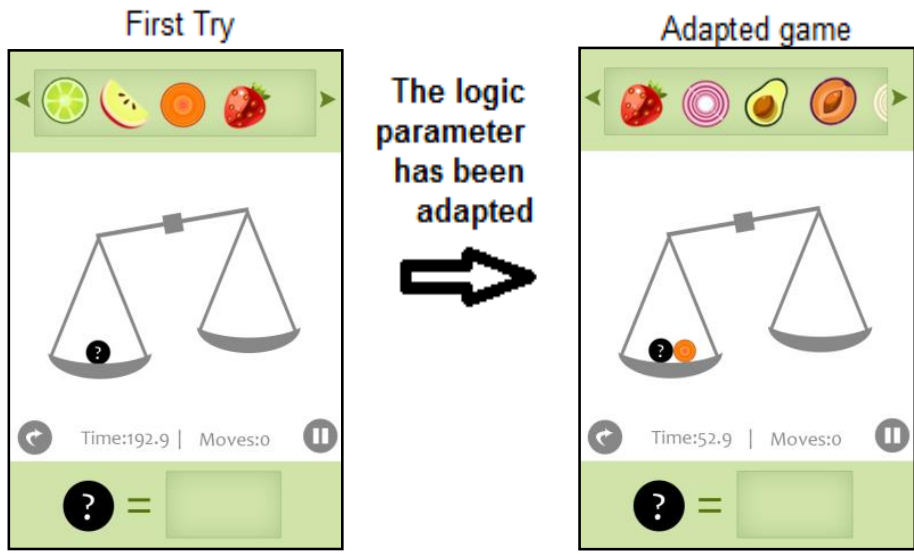

Figure 5. The Adaptation of the EquaFun Serious Game

The data obtained after several uses of the proposed game by several learners "Group 2", have been cultured in three categories by using a Expectation-Maximization [14] algorithm, an algorithm often used in educational data mining to cluster learners' performances, the criteria used to cluster the learners are the criteria already cited above "time spent per equation, total time, right answers, wrong answers, redundant faults", Figure 6.

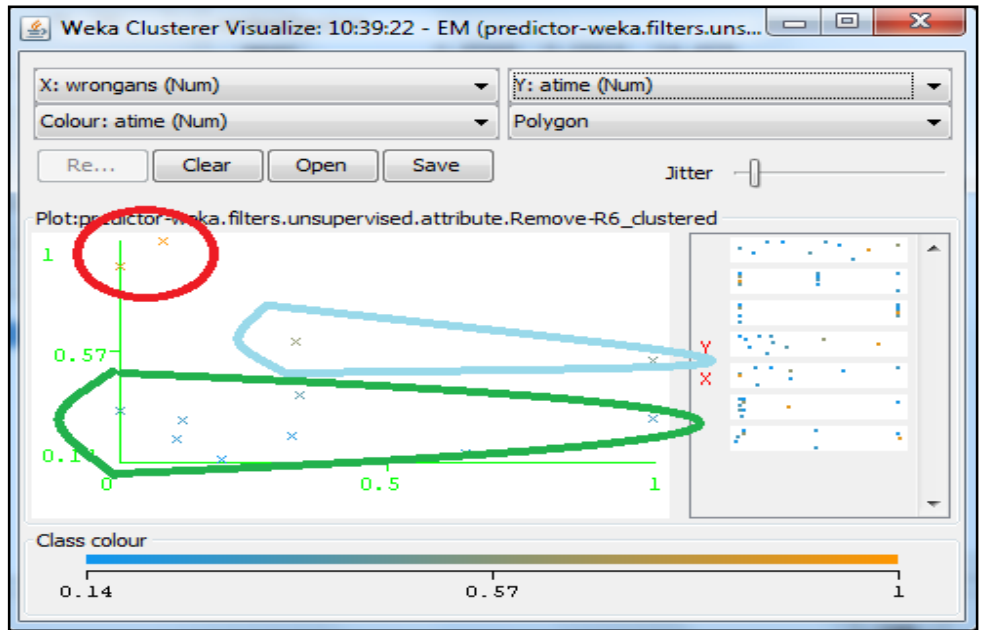

Figure 6. Learning analytics of the learners.

As presented in Figure 6, there are three categories of learners, clustered according to their performances "good, medium and bad". 50\% of the them had good performance; $33 \%$ had medium performance, by cons $17 \%$ had a bad performance, according to the given results the majority of the learners are satisfied and don't have issues concerning resolving mathematical equations, proving that the adaptation of the serious games according the learner performances, has helped them to upgrade their level in the right way without the interaction of the instructor, this proposed manner of learning has proved its effectiveness and can be used in other areas with other parameters and conditions.

Another way to prove the effectiveness of the proposed serious game, and its impact on the learners, is comparing the adapted and the non adapted game. This comparison is based on finding the difference of the learning degree of each group "group 1" the group that use the non adapted version of game and "group 3 " the group that use the adapted version. The Table 4 details this comparison.

Table 4. Comparison of adapted and non adapted Equafun 


\begin{tabular}{ccccc}
\hline & $\begin{array}{c}\text { Average of } \\
\text { the time } \\
\text { spent per } \\
\text { equation }\end{array}$ & $\begin{array}{c}\text { Average of } \\
\text { the right } \\
\text { answers }\end{array}$ & $\begin{array}{c}\text { Average of } \\
\text { the wrong } \\
\text { answers }\end{array}$ & $\begin{array}{c}\text { average of } \\
\text { the redundant } \\
\text { faults }\end{array}$ \\
\hline $\begin{array}{c}\text { None- } \\
\text { Adapted } \\
\text { game } \\
\text { Adapted } \\
\text { game }\end{array}$ & 5,2 & 2,8 & 3,2 & 3,4 \\
\hline
\end{tabular}

As presented in the comparison above, the parameters that have been taken into consideration are "average time spent per equation average of the wrong and right answers and the average of the redundant faults mad by the learners". Those parameters are decisive to measure the learners' performances, have a general view on their improvement, and have an accurate comparison between the two versions of the game. The obtained results show that the adapted game is more beneficial for the learners than the non-adapted. The average values of the adapted game are good compared to the non-adapted.

The four game parameters "reflex, logic, speed and memory" can be adapted positively by incremented their values or negatively by the opposite way. According to the data saved in the database and as presented in the Figure 7, the parameter that has been more changed positively is the logic parameter, then become speed, reflex then memory. By cons the parameter that has been changed negatively "Figure 8 " is logic, speed, and reflex then memory. Which leads to conclude, that the young learners have several difficulties with logical operations against they don't have difficulties with memorization thanks to their fasting age. For this reason experts and pedagogues must think how to improve the logical reasoning of this kind of learners by introducing more fun manners.

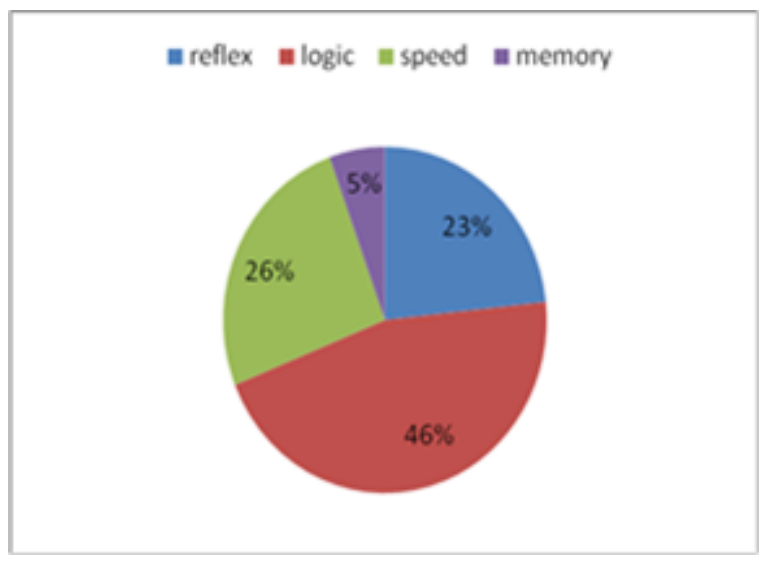

Figure 7. Pie chart of the Postive Adaptation of Paremeters

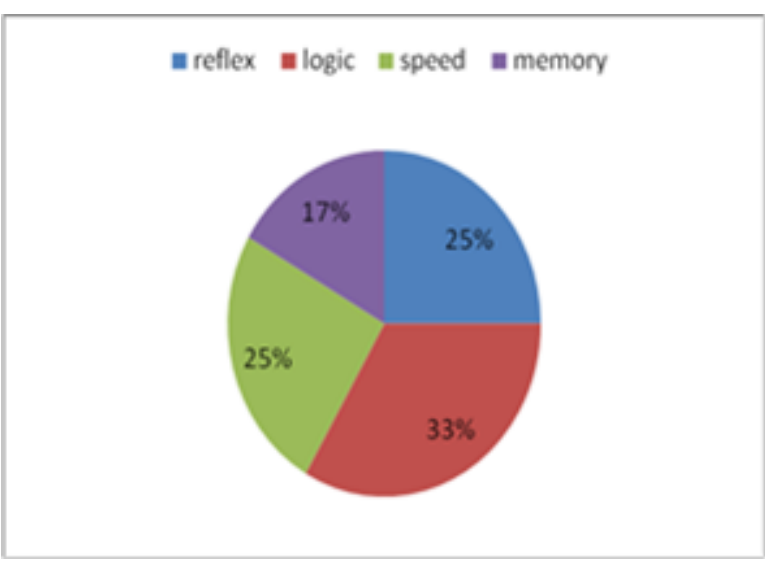

Figure 8. Pie chart of the Negative Adaptation of Paremeters

The comparison of the results obtained by the proposed serious game with a similar game [15] that teaches arithmetic basics, show that both of them are very promising, even if those serious games are very basic and simple, they have received very positive comments. Both of them have improved the learners' math skills with different ways "challenging world with increasing level of difficulty, and enjoyable combat role playing game world". The differences between the two games are the parameters that will be changed in the first one there are reflex, logic, speed and memory. By cons the dynamic difficulty will be changed in the second one.

The adaptation based on the learners' performances has proved its success, according to the obtained results of both learning analytics or the comparison of the tow version of the proposed game. Generally the process of adaptation adopted in their paper, allows students to experience the games, each one according to his level; every experience is different from others; which make it a flexible and beneficial game for intelligent learning. 


\section{CONCLUSION}

The important role that plays serious games in the learning process to improve the leaning capabilities of the learners and allow them to learn new knowledge and skills; will engender a need to have new techniques and tools that will make this kind of video games more effectives and beneficial; among these techniques there is the adaptation process that has been detailed in this paper.

As mentioned before, this process of adaptation based on the learner performance can adapt a variety of game parameters, in order to, improve learners' abilities to learn without the interaction of the instructors. According to the obtained results of the concept adopted in this paper has allowed resolution of several issues encountered by the young learners to resolve mathematical equations. The proposed process of adaptation can be used also to adapt the assessment during the sequence of the game, in order to evaluate the knowledge gained in a more deterministic manner.

\section{REFERENCES}

[1] Sitzmann, T. (2011) A meta-analytic examination of the instructional effectiveness of computer-based.

[2] Wouters, P.J.M., Van der Spek, E.D. and Van Oostendorp, H. (2011) Measuring learning in serious games: a case.

[3] Walker, J. (2009) Left 4 Dead 2: Exclusive RPS Hand on Preview. Rock Paper Shotgun, PC Gaming since 1873. Online available: http://www.rockpapershotgun.com/2009/06/01left-4-dead-2-exclusive-rps-preview.

[4] Brathwaite, B. and Schreiber, I. (2009) Challenges for game designers. Boston, MA: Charles River media.

[5] Bakkes, S. Spronck, P. and van den Herik, J. (2009) Rapid and reliable adaptation of videogame AI. IEEE Transactions Computational Intelligence and AI in Games, 1(2), 93-104.

[6] Roberts, D.L. and Isbell, C.L. (2008) A survey and qualitative analysis of recent advances in drama management. Int. Trans. Sci. Appli., 3(2), 61-75.

[7] Niehaus, J. and Riedl, M.O. (2009) Scenario adaptation: an approach to customizing computer-based training games and simulation. In Proceedings AIED Workshop Intell Edu. Game. Brighton, UK (pp. 89-98).

[8] Christoph Klimmt1, Christopher Blake2, Dorothée Hefner2, Peter Vorderer3, and Christian Roth3, Player Performance, Satisfaction, and Video Game Enjoyment.

[9] Sander Bakkes, Pieter Spronck, and Jaap van den Herik Rapid Adaptation of Video Game AI.

[10] JAVA Agent Development Framework. Available on line: http://jade.tilab.com/ (accessed on 21 October 2014).

[11] McCulloch, W.S. and Pitts, W.H. (1943) A Logical Calculus of the Ideas Immanent in Nervous Activity. Bulletin of Mathematical Biophysics, 5, 115-133.

[12] Martin Riedmiller und Heinrich Braun: Rprop - A Fast Adaptive Learning Algorithm. Proceedings of the International Symposium on Computer and Information Science VII, 1992.

[13] Quinlan, J.R., C4.5: Programs for Machine Learning, Morgan Kaufmann, SanFrancisco, 1993.

[14] Dempster, A.P.; Laird, N.M.; Rubin, D.B. (1977). "Maximum Likelihood from Incomplete Data via the EM Algorithm". Journal of the Royal Statistical Society, Series B 39 (1): 1-38. JSTOR 2984875.

[15] Jonathan Tremblay, Bruno Bouchard, Abdenour Bouzouane. ADAPTIVE GAME MECHANICS FOR LEARNING PURPOSES: making serious games playable and fun, Université du Québec à Chicoutimi, (Québec) (Rolling and Adams, 2003) Canada 555 boul. Université, G7H 2B1. 